\title{
FLUCTUACIÓN ESTACIONAL DE MOSCAS DE LA FRUTA ANASTREPHA SPP. Y CERATITIS CAPITATA (WIEDEMANN, 1824) (DIPTERA: TEPHRITIDAE) EN TRAMPAS MCPHAIL EN PIURA Y EN ICA, PERÚ
}

\author{
Norma NOLASCO' 1 , y José IANNACONE ${ }^{2}$ \\ 1 Laboratorios de Sanidad Vegetal. Servicio Nacional de Sanidad Agraria (SENASA). Av. La \\ Molina 1915, Lima 12, La Molina, LIMA-PERÚ. E-mail: nnolasco@senasa.gob.pe \\ 2 Laboratorio de Ecofisiología Animal. Facultad de Ciencias Naturales y Matemática. \\ Universidad Nacional Federico Villarreal. Calle San Marcos 383, Lima 21, Pueblo Libre, \\ LIMA, PERÚ. E-mail: joseiannacone@yahoo.es
}

\begin{abstract}
RESUMEN
Se realizó un estudio sobre la diversidad, la fluctuación estacional y la proporción sexual de Moscas de la fruta (Tephritidae) entre enero y diciembre del 2002, capturadas con trampas McPhail de plástico en los departamentos de Piura e Ica, Perú. En el Valle Alto Piura y en Ica en cuatro valles (Chincha, Pisco, Ica y Nazca) se evaluó el $n^{\circ}$ de moscas capturadas/trampa/semana, identificándose tres especies en común: Anastrepha distincta Greene, 1934, Anastrepha fraterculus (Wiedemann, 1830), y Ceratitis capitata (Wiedemann, 1824). En Piura se encontró exclusivamente a Anastrepha chiclayae Greene, 1934, Anastrepha obliqua (Macquart, 1835) y Anastrepha striata Schiner, 1868. En cambio en Ica exclusivamente se registró a Anastrepha serpentina (Wiedemann, 1830). Piura presentó una mayor riqueza de tephritidae en comparación a Ica. En Piura, la especie dominante y constante fue $A$. obliqua y en Ica fue $C$. capitata. El índice de diversidad de Shannon-Wiener $\left(\mathrm{H}^{\prime}\right)$ no presentó fluctuaciones estacionales en Piura; en cambio en Ica fue más alto en primavera-verano. Las poblaciones de $A$. fraterculus se incrementaron en el verano en Piura e Ica. En adición, para C. capitata y el total de moscas de la fruta se observó un aumento en verano en Piura y en otoño en Ica. A. obliqua en Piura no presentó fluctuaciones estacionales. Del total de especimenes capturados en Ica, el 66,5\% fueron hembras y el 33,5\% fueron machos. En Piura, el 60,5\% fueron hembras y el 39,5\% fueron machos. La proporción sexual global de macho-hembra fue de 1:1,7 (63,5\% hembras y 36,5\% machos). La temperatura y la presencia de especies frutícolas hospedantes de moscas de la fruta en estado de maduración explican las fluctuaciones observadas.
\end{abstract}

Palabras clave: diversidad, estacionalidad, moscas de la fruta, Perú.

\begin{abstract}
A study on diversity, seasonal fluctuation and sex ratio of fruit-flies (Tephritidae) was performed between January and December, 2002, using with plastic McPhail traps in Piura and Ica state, Peru. At Valle Alto Piura and in four vallies (Chincha, Pisco, Ica and Nazca) at Ica the number of fruit-flies captured trap ${ }^{-1}$ week $^{-1}$ was recorded, and three species in common: Anastrepha distincta Greene, 1934, Anastrepha fraterculus (Wiedemann, 1830), and Ceratitis capitata (Wiedemann, 1824) were identified. In Piura Anastrepha chiclayae Greene, 1934, Anastrepha obliqua (Macquart, 1835) and Anastrepha striata Schiner, 1868 were found exclusively. On the other hand, traps in Ica exclusively captured
\end{abstract}


Anastrepha serpentina (Wiedemann, 1830). Piura presented a higher richness of tephritidae in comparison to Ica. In Piura, the dominant and constant species was $A$. obliqua while in Ica it was $C$. capitata. Shannon-Wiener $\left(\mathrm{H}^{\prime}\right)$ diversity index did not show seasonal fluctuations in Piura; but in Ica its values were higher in spring-summer. A. fraterculus populations increased in Piura and Ica in the summer. In addition, $C$. capitata and fruit-flies in general registered an increase in Piura in the summer and in Ica in the fall. A. obliqua in Piura did not show seasonal fluctuations. Of the total of specimens captured in Ica, $66.5 \%$ were females and $33.5 \%$ were males. In Piura, $60.5 \%$ were females and $39.5 \%$ were males. Global sex ratio of males-females was $1: 1.7$, with $63.5 \%$ females and $36.5 \%$ males. Temperature and ripening host fruit species presence explained diversity and observed fluctuations.

Key Words: diversity, fruit flies, Peru, seasonality.

\section{INTRODUCCIÓN}

Bajo el concepto de "moscas de la fruta", se reconocen a especies de Tephritidae. En el Perú, por lo general se presta mayor atención a Ceratitis capitata (Wiedemann, 1824) y a especies del género Anastrepha Schiner spp., como Anastrepha chiclayae (Greene, 1934), Anastrepha distincta (Greene, 1934), Anastrepha fraterculus (Wiedemann, 1830), Anastrepha obliqua (Macquart, 1835), Anastrepha serpentina (Wiedemann, 1830) y Anastrepha striata (Schiner, 1868) (Korytkowski 1993a,b, 2001).

La mosca del mediterráneo, C. capitata, originaria del Noreste de África (Raga et al. 2006), es una de las plagas de mayor importancia económica del mundo, por varias razones: 1) por sus efectos en la productividad de las plantas hospederas, que pueden ir de 10 a $75 \%$ de reducción en los rendimientos; 2) por las restricciones cuarentenarias que imponen mercados mundiales como América del Norte y Asia Pacífico; 3) por la existencia de una gran variedad y número de hospederos de la plaga en más de 200 frutas distintas; 4) por el impacto en los precios; y 5) por sus externalidades negativas (salud pública, aplicación de plaguicidas y sociales) (Corvalán 2004).

Las moscas de la fruta en el género Anastrepha spp. son nativas del Neotrópico con más de 200 especies descritas, que incluyen plagas de importancia económica de diversos cultivos frutícolas de América Latina, y son consideradas plagas cuarentenarias para muchos países del hemisferio norte (Caraballo 2001, Ibáñez \& Cruz 2001, Jaldo et al. 2001, Korytkwoski 2001, Tejada 2001, Toledo et al. 2001, Robacker et al. 2003, Hernández \& Morales 2004).

La diversidad de climas y zonas ecológicas para la producción hortofrutícola en el Perú, hace propicia la proliferación de las moscas de las Frutas, cuyo ciclo biológico resulta por lo mismo ininterrumpido, a causa de la variedad de hospederos de diferente ciclo vegetativo que fructifican prácticamente a través de todo el año (Velarde \& Esparza 1993). Haciendo un análisis del potencial hortofrutícola en el Perú, podemos mencionar en la costa norte, a Piura y Lambayeque, por presentar las especies de frutales de mayor importancia para fines de exportación, como son mango Mangifera indica L, limón rugoso Citrus jambhiri (Luch) y maracuyá 
Passiflora edulis Sims; en la costa central, a Lima e Ica, con producciones frutícolas de cítricos Citrus spp., mango, manzana Malus domestica Borkh, vid Vitis vinifera L., palto Persea americana Mill y limón y en la costa sur, a los Departamentos de Arequipa, Moquegua y Tacna, que producen olivo Olea europaea L., vid, palto, peral Pyrus communis L. y manzana (Velarde \& Esparza 1993).

Las trampas son dispositivos que atraen a los insectos para capturarlos o destruirlos (Piñero et al. 2002, Raga et al. 2006). Comúnmente se utilizan para detectar la presencia de insectos o para determinar su ocurrencia estacional y su abundancia (Salles 1999), con miras a orientar formas de control. Ocasionalmente, las trampas pueden utilizarse como método directo de destrucción de insectos (Cisneros 1995). El uso de trampas tiene las ventajas de no dejar residuos tóxicos, de operar continuamente, de no ser afectado por las condiciones agronómicas del cultivo y, en muchos casos, de tener un bajo costo de operación. Una limitación en el uso de las trampas es que éstas actúan solamente contra los adultos y no contra las larvas que son las formas en que muchos insectos causan los daños (Cisneros 1995). Para capturar adultos de mosca de la fruta se utiliza la trampa McPhail, cebada con un atrayente de tipo alimenticio (Paxtian et al. 2001, Montoya et al. 2002).

El presente trabajo tuvo como objetivo determinar la diversidad, la fluctuación estacional y la proporción sexual de especies de Anastrepha spp. y C. capitata "Moscas de la fruta" (Diptera: Tephritidae) colectadas en Trampas McPhail en Piura e Ica, Perú, durante 2002.

\section{MATERIAL Y MÉTODOS}

Área de estudio. El estudio se realizó en dos departamentos del Perú, Piura e Ica, cinco zonas de producción o valles, y 13 sectores, entre enero y diciembre del año 2002. Las cinco zonas de producción escogidas presentaron las siguientes características:

Alto Piura: Situado en el Departamento de Piura $\left(4^{\circ}\right.$ y $6^{\circ} \mathrm{LS}$ y $79^{\circ}$ y $\left.81^{\circ} \mathrm{LW}\right)$ a $135 \mathrm{msnm}$. La red estuvo compuesta por 206 trampas McPhail. Los censos se realizaron en un total de 11 semanas no consecutivas a lo largo del 2002 . La temperatura fluctúo entre $22,4^{\circ} \mathrm{C}$ a $29,7^{\circ} \mathrm{C}\left(25,6^{\circ} \mathrm{C} \pm 2,6\right)$.

Chincha: Situado en el Departamento de Ica ubicado a $198 \mathrm{~km}$ al Sur de Lima $\left(12^{\circ} 12^{\prime} 13^{\prime \prime}\right.$ a $13^{\circ} 35^{\prime} 40^{\prime \prime}$ LS y $75^{\circ} 20^{\prime} 02^{\prime \prime}$ a $76^{\circ} 12^{\prime} 06^{\prime \prime}$ LW) a $97 \mathrm{msnm}$. Los censos se realizaron en un total de 32 semanas no consecutivas a lo largo del 2002 . La temperatura fluctúo entre 16,5 y $23^{\circ} \mathrm{C}\left(19,5^{\circ} \mathrm{C} \pm 2,6\right)$.

Pisco: Situado en el Departamento de Ica, ubicado en la parte Central y

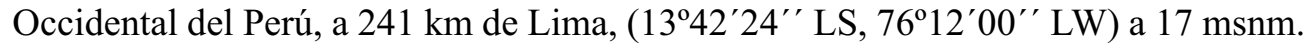
Los censos se realizaron en un total de 28 semanas no consecutivas a lo largo del 2002. La temperatura fluctúo entre 16,6 y $25,1^{\circ} \mathrm{C}\left(20,1^{\circ} \mathrm{C} \pm 2,9\right)$. 
Ica: ubicado a $300 \mathrm{~km}$ al sur de Lima $\left(14^{\circ} 04^{\prime} 00^{\prime \prime} \mathrm{LS}\right.$ y $\left.75^{\circ} 43^{\prime} 24^{\prime \prime} \mathrm{LW}\right)$ a 408 msnm. Los censos se realizaron en un total de 29 semanas no consecutivas a lo largo del 2002. La temperatura fluctúo entre 17,3 y $25,5^{\circ} \mathrm{C}\left(20,9^{\circ} \mathrm{C} \pm 2,5\right)$.

Nazca: ubicado a $447 \mathrm{~km}$ al sur de Lima, a $600 \mathrm{msnm}\left(14^{\circ} 49^{\prime} 44^{\prime \prime}\right.$ LS y $\left.74^{\circ} 56^{\prime} 18^{\prime \prime} \mathrm{LW}\right)$. Los censos se realizaron en un total de 21 semanas no consecutivas a lo largo del 2002. La temperatura se encontró entre $15,8-22,8^{\circ} \mathrm{C}\left(19,1^{\circ} \mathrm{C} \pm 2,6\right)$.

La evaluación se hizo cada siete días por rutas/sectores en las cinco zonas de producción escogidas.

Moscas de la fruta. La muestra de estudio estuvo conformada por 3506 especimenes de "moscas de la fruta" que se identificaron en el Laboratorio de Taxonomía y Sistemática del PNMF (Programa Nacional de Mosca de la Fruta), capturadas en las trampas McPhail de la Red Oficial de Trampeo y Monitoreo de Moscas de la Fruta instaladas en cinco zonas de producción: Valle Alto Piura (SENASA [Servicio Nacional de Sanidad Agraria]-Piura), Chincha, Pisco, Ica y Nazca (SENASA-Ica) durante todo el año 2002 (Tejada 2001, 2002). 1788 especímenes correspondieron a SENASA-Piura y 1718 a SENASA-Ica.

La trampa McPhail consistió en un recipiente de plástico (García \& Corseuil 1998/1999) con una base de color amarillo de $15 \mathrm{~cm}$ de diámetro y $6 \mathrm{~cm}$ de alto, con una invaginación en la parte inferior de $5 \mathrm{~cm}$ de diámetro y 6 de alto, y una parte superior transparente de $9 \mathrm{~cm}$ de alto. Las trampas fueron colocadas a $450 \mathrm{~m}$ de distancia entre una y otra, a una altura de $2 \mathrm{~m}$ y cercanas a plantas que son hospedantes de "mosca de la fruta" (García et al. 2003), generalmente bajo sombra, no rodeadas de follaje muy denso. El atrayente alimenticio colocado por trampa estuvo formado por 10 $\mathrm{mL}$ de proteína hidrolizada, $5 \mathrm{~g}$ de bórax y $235 \mathrm{~mL}$ de agua. Para todos los casos fue determinado el $\mathrm{N}^{\circ}$ de adultos de mosca de la fruta capturados trampa-1 semana-1.

Identificación: los insectos colectados fueron colocados en una placa Petri, conteniendo alcohol al $70 \%$, y con ayuda de pinzas rectas de punta fina y pinzas curvas de punta fina se procedió a observar bajo un Estereoscopio binocular (NikonR, Modelo YS-100, El Segundo, California, USA). Los especimenes identificados fueron separados por grupos en placas Petri cuadradas de $9,5 \mathrm{~cm}$ de lado, según género y especie, siendo a la vez sexados. La identificación se realizó principalmente empleando las claves de Korytkowski (1993a,b, 2001), y complementado con las claves de Foote (1967), Ross et al. (1982), McAlpine (1989), Tejada (2002) y Norrbom (2006). Cuando la identificación de la especie fue dudosa al emplear las claves, se procedió a hacer montaje de alas y de genitalias externas (masculinas y femeninas).

Análisis de datos. Con el objetivo de verificar la constancia de cada especie de mosca de la fruta en los cinco valles evaluados, fue determinado el índice de constancia (Sampaio et al. 2002). Las especies fueron consideradas constantes (C) cuando fueron registrados en más del $50 \%$ de las semanas, comunes (c) cuando se presentaron entre un 10 y $50 \%$, y finalmente raros (r) hasta en un $10 \%$ de las semanas 
evaluadas. El Índice de diversidad de Shannon-Wienner [H'] se determinó siguiendo el procedimiento de Moreno (2001).

Se determinó la correlación existente entre la altitud de cada zona de producción evaluada, el $\mathrm{N}^{\circ}$ de moscas total capturada trampa ${ }^{-1}$ semana $^{-1}$, la temperatura y la riqueza de especies por localidad. Se realizó un ANDEVA, para demostrar si existían diferencias significativas estacionales (Primavera-Verano, Otoño e Invierno para el departamento de Ica, y Verano, Otoño-Invierno y primavera para Piura) entre la temperatura, $\mathrm{N}^{\circ}$ de adultos trampa ${ }^{-1}$ semana-1 de $A$. distincta, $A$. fraterculus, $A$. serpentina, $A$. chiclayae, $A$. striata, A. obliqua, C. capitata, frecuencia total de Tephritidae y H'. En adición se empleó el ANDEVA para determinar si existían diferencias en la temperatura durante el 2002 entre los cinco valles evaluados. Se utilizó el paquete estadístico SPSS versión 12,0 para windows 98,0 para el cálculo de los estadísticos descriptivos e inferenciales (SPSS 2003). Se determinó la significancia al $5 \%$.

\section{RESULTADOS Y DISCUSIÓN}

Entre los meses de enero a diciembre del 2002 se colectaron 3.506 especimenes de Anastrepha y Ceratitis capturadas en Trampas McPhail en los Departamentos de Ica y Piura. 1.718 especimenes se obtuvieron del departamento de Ica en las zonas de producción de Chincha, Pisco, Ica y Nazca, y 1.788 especimenes en el Valle Alto Piura (Departamento de Piura).

Diversidad de adultos en el departamento de Ica. En el departamento de Ica, de los 1.718 especimenes de "moscas de la fruta" capturados, 149 fueron de Chincha, 1.068 de Pisco, 428 de Ica y 72 en Nazca, es decir, 8,7\%, 62,2\%, 24,9\% y 4,2\%, respectivamente. Se identificaron cuatro especies de "moscas de la fruta" (Cuadro 1). El N ${ }^{\circ}$ moscas capturadas trampa ${ }^{-1}$ semana $^{-1}$ y el porcentaje de ocurrencia presentó el siguiente orden decreciente: $C$. capitata $>A$. distincta $>A$. fraterculus $>$ A.serpentina (Cuadro 1).

La mayor captura correspondió a la zona de producción de Pisco $(38,1$ moscas trampa $^{-1}$ semana $\left.^{-1}\right)$. C. capitata fue la especie que predominó en este valle. La menor captura correspondió a la zona de producción de Nazca $\left(3,45\right.$ moscas trampa ${ }^{-1}$ semana $^{-1}$ ) (Cuadro 1). En este valle se registró la menor diversidad de cultivos hospederos. Por otro lado la mayor riqueza específica $(n=4)$ se presentó en la zona de producción de Chincha (Cuadro 1), debido a que solo en este valle se tuvo al lúcumo Lucuma obovata $\mathrm{Hbk}$, especie frutícola hospedera de A. serpentina (PNMF 2001). Sin embargo, A. serpentina fue la especie de menor predominancia, debido a su especificidad por la lúcuma, que cuenta con una mínima cantidad de área sembrada en Chincha. Ceratitis capitata fue la especie predominante en las zonas de producción de Pisco, Ica y Nazca, debido posiblemente a que las temperaturas oscilaron entre $19,1^{\circ} \mathrm{C}$ y $20,9^{\circ} \mathrm{C}$ fueron adecuadas para su desarrollo y menores a las temperaturas óptimas para algunas especies de Anastrepha (Arellano 2001). 


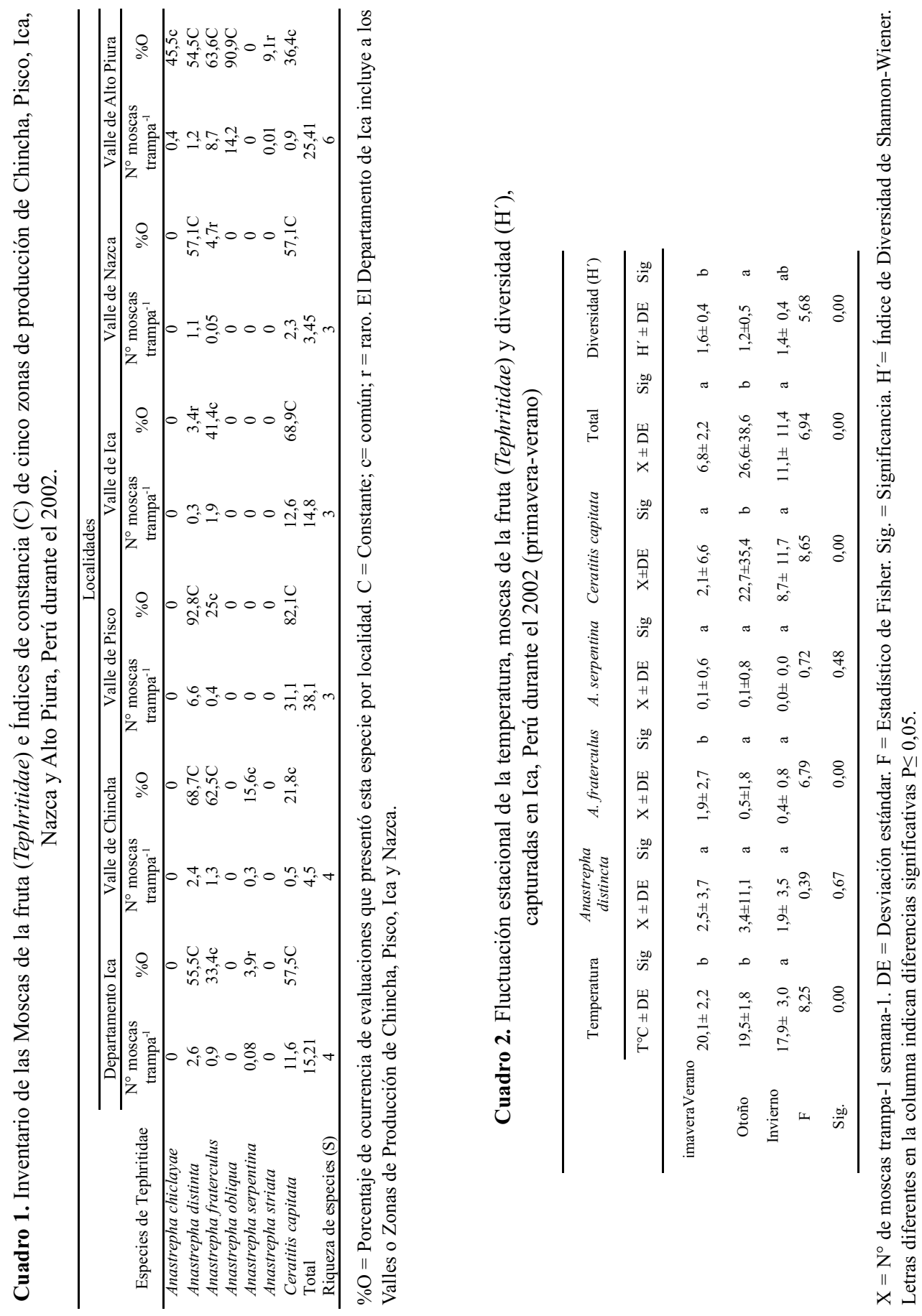


Fluctuación estacional de adultos en el Departamento de Ica. En el departamento de Ica, la temperatura fue menor en invierno en comparación con primavera-verano y otoño (Cuadro 2). La mayor captura de $A$. fraterculus correspondió a primavera-verano, estaciones con las más altas temperaturas. García et al. (2003) encontró una relación positiva entre mayores niveles de A. fraterculus y altas temperaturas. Anastrepha fraterculus es una especie multivoltina, con seis a once generaciones al año, polífaga, siendo conocida en más de 80 hospederos y 18 familias de plantas (Jaldo et al. 2001, García et al. 2003, Oroño et al. 2005). El $\mathrm{N}^{\circ}$ moscas capturadas trampa ${ }^{-1}$ semana $^{-1}$ fue mayor en otoño para $C$. capitata y para el total de moscas de la fruta (Cuadro 2). Anastrepha distincta y A. serpentina, no presentaron estacionalidad (Cuadro 2). El índice de diversidad $\left(\mathrm{H}^{\prime}\right)$ presentó un mayor valor en primavera-verano, el cual fue diferente al índice $\mathrm{H}^{\prime}$ del otoño (Cuadro 2).

Según el Censo Frutícola del Departamento de Ica, Perú del año 2002, los cultivos más importantes son mango, ciruelo Spondias purpurea L. y cítricos, los cuales son hospederos principales de $A$. fraterculus y C. capitata (Ovruski et al. 2003). En Ica, la maduración del durazno Prunus persica (L.) Batsch, del higo Ficus carica L. y del mango se inicia entre finales de primavera y verano, mientras que la del pacae Inga feullei D.C. se da a fines del verano y en el otoño, seguido de los cítricos [naranja dulce Citrus sinensis (L.) Osbeck, pomelo Citrus aurantium L., tangelo Citrus reticulata L. x Citrus paradisi MacFadyn, naranja china Fortunella spp., mandarina Citrus spp. y limón rugoso] a fines de otoño y durante el invierno. A fines del invierno e inicio de primavera no hay maduración de los hospedantes, pero existen los frutos caídos. Por ende, se observa a lo largo de todo el año la presencia de frutas hospederos de mosca de la fruta (Sivinski et al. 2004). Esto permite a las diversas especies de moscas de la fruta mantenerse presentes en el campo (Cuadro 2). La disponibilidad estacional de los frutos hospederos se encuentra entre los principales factores que actúan sobre la densidad de las moscas de la fruta (García et al. 1998/1999, Uchóa et al. 2002).

Diversidad de adultos en el departamento en Piura. De los 1788 especimenes de moscas de la fruta capturados en el departamento de Piura: 619 especimenes correspondieron a Yapatera, 591 especimenes a Campanas y 578 especimenes a La Matanza (35\%, $33 \%$ y $32 \%)$ respectivamente. No se observaron diferencias en el $\mathrm{N}^{\circ}$ moscas capturadas trampa ${ }^{-1}$ semana $^{-1}$ entre los tres sectores, debido a que dichas zonas geográficas limitan entre sí y las condiciones climáticas y los frutales hospederos son similares (MINAGR 2002). En Valle Alto Piura, se identificaron seis especies de "moscas de la fruta", cinco pertenecen al género Anastrepha y $C$. capitata (Cuadro 1). El $\mathrm{N}^{\circ}$ moscas capturadas trampa ${ }^{-1}$ semana $^{-1}$ en orden decreciente por especie fue: $A$. obliqua $>$ A. fraterculus $>A$. distincta $>C$. capitata $>$ A. chiclayae $>A$. striata. Con relación al porcentaje de ocurrencia se observó la siguiente secuencia en orden descendente: A. obliqua $>$ A. fraterculus $>$ A. distincta $>$ A. chiclayae $>$ C. capitata $>$ A. striata (Cuadro 1). 
Fluctuación estacional de adultos en el Departamento de Piura. En Piura, se encontraron diferencias estacionales en la temperatura, otoño-invierno $>$ primavera $>$ verano (Cuadro 3). El $\mathrm{N}^{\circ}$ de moscas capturadas trampa ${ }^{-1}$ semana $^{-1}$ fue mayor en otoño-invierno para $A$. distincta, y en verano para $A$. fraterculus y para $C$. capitata (Cuadro 3). No se encontraron diferencias estacionales en el $\mathrm{N}^{\circ}$ moscas capturadas trampa-1 $^{-1}$ semana-1 en $A$. chiclayae, A. oblicua y en los valores de $\mathrm{H}^{\prime}$ (Cuadro 3). En adición, el $\mathrm{N}^{\circ}$ total de moscas capturadas trampa ${ }^{-1}$ semana $^{-1}$ en trampas McPhail en el Valle Alto Piura, fue mayor en el verano (Cuadro 3). Esto es debido a que en estos meses está en maduración $M$. indica "mango", hospedero principal de A. obliqua y A. fraterculus (Sivinski et al., 2004), mientras que la menor captura correspondió al otoño-invierno, estación en la que está en maduración el plátano o banano, y el limón (cultivos de mayor área cultivada en Valle Alto Piura), pero poco preferidas para ambas especies de Anastrepha.

También se puede observar que el $\mathrm{N}^{\circ}$ moscas totales capturadas trampa-1 semana1 en otoño-invierno disminuye, ya que en estos meses no está el mango en etapa fenológica de maduración (Cuadro 3). Dos Santos et al. (1998) y Sivinski et al. (2004) señalan que la fenología de la planta hospedera influye en las fluctuaciones de Anastrepha. En Piura, la maduración del manzano, mango, mandarina, limón, durazno, vid y plátano ocurre en verano. Durante el otoño-invierno, los cítricos (mandarina, naranjo y limón rugoso), el olivo y el maracuyá se encuentran en maduración. A inicio de primavera hay maduración del durazno y el mango. Lo que permite a las diversas especies de moscas de la fruta mantenerse presentes en el campo (Cuadro 3).

Cuadro 3. Fluctuación estacional de la temperatura, moscas de la fruta (Tephritidae) y diversidad $\left(\mathrm{H}^{\prime}\right)$, capturadas en el Valle Alto Piura, Piura, Perú durante el 2002.

\begin{tabular}{|c|c|c|c|c|c|c|c|c|c|c|c|c|c|c|c|c|}
\hline & \multicolumn{2}{|c|}{ Temperatura } & \multicolumn{2}{|c|}{$\begin{array}{l}\text { Anastrepha } \\
\text { distincta }\end{array}$} & \multicolumn{2}{|c|}{ A. fraterculus } & \multicolumn{2}{|c|}{ A. chiclayae } & \multicolumn{2}{|c|}{ A. obliqua } & \multicolumn{2}{|c|}{$\begin{array}{l}\text { Ceratitis } \\
\text { capitata }\end{array}$} & \multicolumn{2}{|l|}{ Total } & \multicolumn{2}{|c|}{ Diversidad $\left(\mathrm{H}^{\prime}\right)$} \\
\hline & $\mathrm{T}^{\circ} \mathrm{C} \pm \mathrm{DE}$ & Sig & $\mathrm{X} \pm \mathrm{DE}$ & Sig & $\mathrm{X} \pm \mathrm{DE}$ & Sig & $\mathrm{X} \pm \mathrm{DE}$ & Sig & $\mathrm{X} \pm \mathrm{DE}$ & Sig & $\mathrm{X} \pm \mathrm{DE}$ & Sig & $\mathrm{X} \pm \mathrm{DE}$ & Sig & $\mathrm{H}^{\prime} \pm \mathrm{DE}$ & Sig \\
\hline Verano & $28,8 \pm 1,0$ & $\mathrm{c}$ & $0,6 \pm 0,6$ & $\mathrm{ab}$ & $7,7 \pm 5,5$ & $\mathrm{~b}$ & $0,3 \pm 0,4$ & $\mathrm{a}$ & $5,4 \pm 3,2$ & $\mathrm{a}$ & $1,0 \pm 1,2$ & $\mathrm{~b}$ & $15,1 \pm 7,2$ & $\mathrm{~b}$ & $1,7 \pm 0,2$ & $\mathrm{a}$ \\
\hline $\begin{array}{l}\text { Otoño } \\
\text { nvierno }\end{array}$ & $23,2 \pm 0,4$ & $\mathrm{a}$ & $1,6 \pm 2,9$ & $\mathrm{~b}$ & $1,8 \pm 1,5$ & $\mathrm{a}$ & $0,08 \pm 0,1$ & $\mathrm{a}$ & $0,1 \pm 0,1$ & a & $0,0 \pm 0,0$ & $\mathrm{a}$ & $3,7 \pm 4,2$ & $\mathrm{a}$ & $2,0 \pm 0,2$ & $\mathrm{a}$ \\
\hline rimavera & $25,5 \pm 1,1$ & $\mathrm{~b}$ & $0,05 \pm 0,1$ & $\mathrm{a}$ & $1,1 \pm 2,4$ & a & $0,07 \pm 0,2$ & $\mathrm{a}$ & $7,1 \pm 7,3$ & $\mathrm{a}$ & $0,04 \pm 0,1$ & $\mathrm{a}$ & $8,4 \pm 6,8$ & $\mathrm{ab}$ & $1,6 \pm 1,1$ & $\mathrm{a}$ \\
\hline $\mathrm{F}$ & 47,6 & & 3,46 & & 9,46 & & 2,49 & & 2,34 & & 6,10 & & 4,81 & & 1,03 & \\
\hline Sig. & 0,00 & & 0,04 & & 0,001 & & 0,10 & & 0,11 & & 0,007 & & 0,01 & & 0,37 & \\
\hline
\end{tabular}

$\mathrm{X}=\mathrm{N}^{\circ}$ de moscas trampa-1 semana-1. $\mathrm{DE}=$ Desviación estándar. $\mathrm{F}=$ Estadístico de Fisher. Sig. $=$ Significancia. $\mathrm{H}^{\prime}=$ Índice de Diversidad de Shannon-Wiener. Letras diferentes en la columna indican diferencias significativas $\mathrm{P} \leq 0,05$. 
Anastrepha obliqua y A. fraterculus son las especies predominantes en el Valle de Piura con $45,6 \%$ y $41,3 \%$ del total de moscas capturadas, respectivamente. Esto es debido a que en esta zona se cuenta con el cultivo de mango $M$. indica y ciruela $S$. purpurea, ambas especies hospederas de estas moscas de la fruta (Hernández \& Morales 2004, Segura et al. 2006). El género predominante es Anastrepha con 1.671 (93,5\% del total) individuos (A. obliqua con 816; A. fraterculus con 738; A. distincta con $62 ; A$. chiclayae con 53 y A. striata con 2 especimenes respectivamente), mientras que Ceratitis presentó la menor captura con 117 especimenes $(6,5 \%$ del total). En Piura, la temperatura es elevada $\left(25,6 \pm 2,6^{\circ} \mathrm{C}\right)$, lo que favorece al complejo Anastrepha, más no así a C. capitata (Arellano 2001).

Proporción sexual de moscas de la fruta en Ica y Piura. Del total de especimenes capturados en Ica, el 66,5\% $(\mathrm{n}=1142)$ son hembras y el $33,5 \%(\mathrm{n}=$ 576) son machos. En el Valle Alto Piura - Piura, las hembras son 1.082 individuos mientras que los machos son 706 , es decir, el $60,5 \%$ son hembras y el $39,5 \%$ son machos. Esto nos indica una proporción global de macho-hembra de 1:1,7. Esto puede deberse a que cuando un espécimen macho finaliza el proceso de cópula, intenta copular con otras hembras. En cambio, la hembra se dedica primero a la búsqueda de alimento, porque requiere de sustancias nutritivas que le ayuden en la reproducción y luego busca un sustrato adecuado para la oviposición (Aluja 1993), es por esto que más hembras fueron colectadas en la trampa McPhail, debido a que contiene proteína hidrolizada, el cual es un atrayente alimenticio (Celedonio 1997).

Análisis comparativo entre los departamentos de Ica y de Piura. Existieron diferencias significativas en la temperatura de las cuatro zonas de Ica: Chincha (19,5 $\left.\pm 2,6^{\circ} \mathrm{C}\right)$, Pisco $\left(20,2 \pm 2,9^{\circ} \mathrm{C}\right)$, Ica $\left(20,9 \pm 2,5^{\circ} \mathrm{C}\right)$, Nazca $\left(19,1 \pm 2,6^{\circ} \mathrm{C}\right)$ en comparación a Piura $\left(25,6 \pm 2,6^{\circ} \mathrm{C}\right)(\mathrm{F}=11,8 ; \mathrm{P}=0,000)$. Solo se observó una relación positiva entre la temperatura y la riqueza de especies $(\mathrm{r}=0,88 ; \mathrm{P}=0,04)$. Por ende, la mayor temperatura en Piura, sería uno de los factores que explicarían la mayor riqueza específica de moscas de la fruta $(\mathrm{n}=6)$ en esta localidad (Cuadro 1).

No se encontró ninguna relación entre la altitud de cada zona de producción evaluada, el $\mathrm{N}^{\circ}$ de moscas total capturada trampa ${ }^{-1}$ semana $^{-1}$, la temperatura y la riqueza de especies por localidad $(\mathrm{P}>0,05)$. Hernández \& Morales (2004) encontraron a $A$. fraterculus en Venezuela en localidades superiores a $1000 \mathrm{~m}$, mientras que por debajo de este rango los registros fueron escasos. En adición, tampoco se encontró una correlación entre el $\mathrm{N}^{\circ}$ de semanas evaluadas por zona de producción y el $\mathrm{N}^{\circ}$ promedio de moscas capturadas trampa-1 semana $^{-1}(\mathrm{r}=0,25 ; \mathrm{P}=$ $0,68)$.

Agradecimientos: Este trabajo fue presentado en dos partes a dos eventos: 1) en la XLV Convención Nacional de Entomología, 01 al 05 diciembre del 2003, Ayacucho, Perú; y 2) en el I Congreso Nacional Estudiantil en Ciencias Ambientales, del 19 al 23 de abril del 2004, Lima, Perú. 


\section{LITERATURA CITADA}

Aluja, M. 1993. Manejo Integrado de la Mosca de la Fruta. 1ra ed. Editorial Trillas. México. 251 pp. Arellano, G. 2001. Evaluación de plagas en café, papayo, piña, plato, plátano y cítricos en Chanchamayo y Satipo. Tesis Magister Scientiae. Universidad Nacional Agraria La Molina. Lima, Perú. 194 pp.

Celedonio, H. 1997. Fluctuación poblacional de adultos de moscas de la fruta en huertos frutales de diversas especies. Programa Moscamed DGSV-SAGAR. Tapachula, Chiapas, México. 213 pp.

Cisneros, V. 1995. Control de Plagas Agrícolas. 2da ed. Editorial Full Print s.r.l. Lima, Perú. 313 pp.

Caraballo, J. 2001. Diagnosis y clave pictórica para las especies del género Anastrepha Schiner, 1868 (Diptera: Tephritidae) de importancia económica en Venezuela. Entomotropica 16:157-164.

Corvalan, L.L. 2004. Evaluación del índice 0,01 capturas/trampa/día como indicador de baja prevalencia de Ceratitis capitata (Wiedemann) en duraznos importados. Agric. Téc. (Chile) 64:82-88.

Dos Santos, J.D., J.H.R. Dos Santos, E.A. Cardoso, R.P. De Souza, M.A. Filgueira \& J.W.N. Chaves. 1998. Population fluctuation of Anastrepha fruitfly species on Guava (Psidium guajava L.) in Mossoró-RN-Brazil. Caatinga 11:91-93.

Foote, R.H. 1967. Tephritidae (Trypetidae, Trupaneidae). Pp. 1-91. In P.E. Vanzolini and N. Papavero (eds). A Catalogue of the Diptera of the Americas. Departamento de Zoología Sección de Agricultura, Sao Paulo, 57. Sao Paulo, Brazil.

García, F.R.M. \& E. Corseuil. 1998/1999. The influence of climatic factors on fruit-flies (Diptera: Tephrtidae) on peach orchards in Porto Alegre, Rio Grande do sul. Uruguaiana 5/6:71-75.

García, F.R.M., J.V. Campos \& E. Corseuil. 2003. Flutuação populacional de Anastrepha fraterculus (Wiedemann, 1830) (Diptera: Tephritidae) na Região Oeste de Santa Catarina, Brasil. Rev. Brasil. Entomol. 47:415-420.

Hernández, O. V. \& V.P. Morales. 2004. Distribución geográfica y plantas hospederas de Anastrepha fraterculus (Diptera: Tephritidae). Folia Entomol. Mex. 43:181-189.

Ibañez, L. A. \& L.L. Cruz. 2001. Glándulas salivales de Anastrepha obliqua (Macquart) (Diptera: Tephritidae): análisis químico y morfológico y actividad biológica de los componentes volátiles. Folia Entomol. Mex. 40:221-231.

Jaldo, H.E., M.V. Gramajo \& E. Willink. 2001. Mass rearing of Anastrepha fraterculus (Diptera: Tephritidae): a preliminary strategy. Florida Entomol. 84:716-718.

Korytkowski, C.A. 1993a. Manual de Identificación de Moscas de la Fruta. Parte II: Genéro Anastrepha Schiner, 1868. Universidad de Panamá. Panamá. 102 pp.

1993b. Manual de Identificación de Moscas de la Fruta. Parte I. Generalidades sobre Clasificación y Evolución de Acalyptratae, Familias: Neriidae, Ropalomeridae, Lonchaeidae, Richardiidae, Otitidae y Tephritidae. Universidad de Panama. Panamá. 94 pp.

2001. Situación actual del género Anastrepha Schiner, 1868 (Díptera: Tephritidae) en el Perú. Rev. per. Entomol. 42:97-158.

McAlpine, J.F. 1989. Phylogeny and classification of the Muscomorpha. Pp. 1397-1518. In: J.F. McAlpine and D.M. Wood (Eds). Manual of Nearctic Diptera Vol. 3. Res. Branch Agr. Canada Monograph 32. Canada.

Ministerio de Agricultura (MINAGR). 2002. Manual de Identificación taxonómica. Especies de Anastrepha frecuentes en trampas McPhail. Servicio Nacional de Sanidad Agraria (SENASA). Programa Nacional de Moscas de la Fruta. Lima, Perú. 54 pp.

Montoya, P., H. Celedonio, H. Miranda, J. Paxtian \& D. Orozco. 2002. Evaluación de sistemas de trampeo y atrayentes para la captura de hembras de Ceratitis capitata (Wied.) y otras moscas de la fruta (Diptera: Tephritidae) en la Región del Soconusco, Chiapas. Folia Entomol. Mex. 41:359-374. 
Moreno, C.E. 2001. Métodos para medir la biodiversidad. Manuales y Tesis SEA. Sociedad Entomológica Aragonesa Madrid, España. 80 pp.

Norrbom, A.L. 2006. Fruit fly (Diptera: Tephritidae) classification \& diversity. Available at http://www.sel.barc.usda.gov//diptera/tephriti/Tephclas.htm. Accessed 10 April 2006.

Oroño, L.E., S.M. Ovruski, A.L. Norrbom, P. Schliserman, C. Colin \& C.B. Martin. 2005. Two new native host plant records for Anastrepha fraterculus (Diptera: Tephritidae) in Argentina. Florida Entomol. 88:228- 232.

Ovruski, S., P. Schliserman \& M. Aluja. 2003. Native and introduced host plants of Anastrepha fraterculus and Ceratitis capitata (Diptera: Tephritidae) in Northwestern Argentina. J. Econ. Entomol. 96:1108-1118.

Paxtian, J., J. Toledo, P. Liedo, A. Oropeza \& R. González. 2001. Captura de Anastrepha ludens (Loew) (Diptera: Tephrititdae) utilizando tres tipos de trampas y cuatro fuentes de atracción. Folia Entomol. Mex. 40:423-426.

Piñero, J., M. Aluja, M. Equihua \& M.M. Ojeda. 2002. Feeding history, age and sex influence the response of four economically important Anastrepha species (Diptera: Tephritidae) to human urine and hydrolyzed protein. Folia Entomol. Mex. 41:283-298.

Programa Nacional de Moscas de la Fruta (PNMF). 2002. Especies de moscas de la fruta y distribución en el Perú. Información para Análisis de Riesgo de Plagas para frutos de Cítricos del Perú. Servicio Nacional de Sanidad Agraria (SENASA). Lima, Perú. 135 pp.

Raga, A., R.A. Machado, W. Dinardo \& P.C. Strikis. 2006. Eficacia de atrativos alimentares na captura de moscas-das-frutas em pomar de citros. Bragantia 65:337-345.

Robacker, D.C., R.L. Mangan, D.S. Moreno \& A.M.T. Moreno. 2003. Behavior and interactions of wild Anastrepha ludens Loew (Diptera: Tephritidae) males on a grapefruit tree. Folia Entomol. Mex. 42:221-237.

Ross, H.H., C.A. Ross \& J.R.P. Ross. 1982. A Textbook of Entomology. 4th ed. John Wiley \& Sons, New York, USA. 666 pp.

Salles, L.A. 1999. Efeito de envelhecimento e da decomposição do atrativo na captura de adultos de Anastrepha fraterculus (Wied.) (Diptera: Tehritidae). Rev. Bras. Agrociencia 5:147-148.

Sampaio, E.V., O. Rocha, T. Matsumura-Tundisi \& J.G. Tundisi. 2002. Composition and abundance of zooplancton in the limnetic zone of seven reservoirs of the Paranapema River, Brazil. Brazilian J. Biol. 62:525-545.

Segura, D.F., T. Vera, C. Cagnotti, N. Vaccaro, O. de Coll, S. Ovruski \& J.L. Cladera. 2006. Relative abundance of Ceratitis capitata and Anastrepha fraterculus (Diptera: Tephritidae) in diverse host species and localities of Argentina. Ann. Entomol. Soc. Am. 99:70-83.

Sivinski, J., M. Aluja, J. Piñero \& M Ojeda. 2004. Novel analysis of spatial and temporal patterns of resource use in a group of tephritid flies of the genus Anastrepha. Ann. Entomol. Soc. Am. 97:504512.

SPSS. 2003. Statistical Package for the Social Sciences. Base 12.0 user's guide: SPSS Inc., Chicago Illinois, USA.

Tejada, G. H. 2001. La red de trampas para vigilancia preventiva de especies exóticas de mosca de la fruta: importante aporte del SENASA a la sanidad Vegetal. Pp. 118. In XLIII Convención Nacional de Entomología. Huancayo, Perú. 4-8 de Noviembre del 2001. Sociedad Peruana de Entomología. Huancayo, Perú.

2002. Manual de Identificación Taxonómica. Especies de Anastrepha más frecuentes e trampas McPhail. MINAG-SENASA. Programa Nacional de Mosca de la Fruta. Lima, Perú. 56 pp.

Toledo, J., M.E. Bustos \& P. Liedo. 2001. Irradicación de naranjas infestadas por Anastrepha ludens (Loew) (Diptera: Tephritidae) como tratamiento cuarentenario. Folia Entomol. Mex. 40:283-295. 
Nolasco \& Iannacone. Moscas de la fruta en Perú

Uchóa-Fernández, M.A., I. Oliveira, R.M.S. Molina \& R.A. Zucchi. 2002. Species diversity of frugivorous flies (Diptera: Tephritoidea) from hosts in the cerrado of the State of Mato Grosso do Sul, Brazil. Neotrop. Entomol. 31:515-524.

Velarde, R. O. \& D.J. Esparza. 1993. Programa Nacional de Prevención, Control y Erradicación de las Moscas de las Frutas en los Valles de la Costa Peruana con Potencial para la Producción y la Exportación Hortofrutícola. IICA. Lima, Perú. 89 pp.

Recibido: 19 de octubre de 2007

Aceptado: 12 de abril de 2008 